\title{
Viscoelastic Effects on Free Convective Three-Dimensional Flow with Heat and Mass Transfer
}

\author{
Rita Choudhury ${ }^{1}$ and Utpal Jyoti Das ${ }^{2}$ \\ ${ }^{1}$ Department of Mathematics, Gauhati University, Assam, Guwahati 781014, India \\ ${ }^{2}$ Department of Mathematics, Rajiv Gandhi University, Rono Hills, Arunachal Pradesh, Itanagar 791112, India
}

Correspondence should be addressed to Utpal Jyoti Das, utpaljyotidas@yahoo.co.in

Received 12 September 2011; Accepted 4 October 2011

Academic Editors: G. Bella and Z. Guo

Copyright ( $) 2012$ R. Choudhury and U. J. Das. This is an open access article distributed under the Creative Commons Attribution License, which permits unrestricted use, distribution, and reproduction in any medium, provided the original work is properly cited.

A theoretical study of free convective three-dimensional heat and mass transfer flow of a viscoelastic fluid along a steadily moving porous vertical plate in presence of transverse sinusoidal suction velocity distribution, and uniform free stream velocity has been considered. The flow becomes three dimensional due to this suction velocity. The governing equations of the flow field are solved by using series expansion method, and the expressions for velocity field, temperature field, skin friction, heat flux in terms of Nusselt number, and mass flux in terms of Sherwood number are obtained. The effects of the viscoelastic parameter on velocity profiles and shear stress with the combination of the other flow parameters are discussed graphically.

\section{Introduction}

The study of combined heat and mass transfer problems with chemical reaction is of great practical importance to engineers and scientists because of its almost universal occurrence in many branches of science and engineering. Such phenomenon is observed in buoyancy-induced motions in the atmosphere, in bodies of water, quasisolid bodies such as earth, and so on. In nature and industrial applications, many transport processes exist where the heat and mass transfer takes place simultaneously as a result of combined effects of thermal diffusion and diffusion of chemical species. In addition, the phenomenon of heat and mass transfer is also encountered in chemical processes industries such as food processing and polymer production. Soundalgekar and Warve [1] have analyzed two-dimensional unsteady free convection flow, past an infinite vertical plate with oscillating wall temperature and constant suction. Lin and $\mathrm{Wu}[2]$ have analyzed the problem of simultaneous heat and mass transfer with entire range of buoyancy ratio for most practical chemical species in dilute and aqueous solutions. Muthucumaraswamy et al. [3] studied the heat and mass transfer effects on flow past an impulsively started infinite vertical plate.
Many research workers are doing investigation of the problem of laminar flow control due to its importance in the field of aeronautical engineering, in view of its applications to reduce drag and enhance the vehicle power requirement by a substantial amount. Initially this subject has been developed by Lachmann [4]. Theoretical and experimental investigations have shown that the transition from laminar to the turbulent flow, which causes the drag coefficient to increase, may be prevented by suction of the fluid and heat and mass transfer from boundary to the wall.

Singh et al. [5] studied the effect on wall shear stress and heat transfer of the flow caused by the periodic suction velocity perpendicular to the flow direction when the difference between the wall temperature and the free stream temperature gives rise to buoyancy force in the direction of the free stream. The effect of the porous medium on the three-dimensional Couette flow and heat transfer was presented by Singh and Sharma [6]. Chaudhary and Sharma [7] studied the three-dimensional convection flow through a porous medium and estimated the effect in heat and mass transfer. Ahmed [8] has studied the effects of heat and mass transfer on the steady three-dimensional flow of a viscous incompressible fluid along a steadily moving porous vertical plate subjected to a transverse sinusoidal velocity. 
The present paper is concerned with the free convective three-dimensional heat and mass transfer flow of viscoelastic incompressible fluid characterized by second-order fluid along a steadily moving porous vertical plate in presence of transverse sinusoidal suction velocity distribution and uniform free stream velocity.

The constitutive equation for the incompressible secondorder fluid is

$$
\sigma=-p I+\mu_{1} A_{1}+\mu_{2} A_{2}+\mu_{3}\left(A_{1}\right)^{2}
$$

where $\sigma$ is the stress tensor, $p$ is hydrostatic pressure, $I$ is unit tensor, $A_{n}(n=1,2)$ are the kinematic Rivlin-Ericksen tensors, and $\mu_{1}, \mu_{2}$, and $\mu_{3}$ are the material coefficients describing the viscosity, elasticity, and cross-viscosity, respectively. The material coefficients $\mu_{1}, \mu_{2}$, and $\mu_{3}$ are taken constants with $\mu_{1}$ and $\mu_{3}$ as positive and $\mu_{2}$ as negative (Markovitz and Coleman [9]). Equation (1) was derived by Coleman and Noll [10] from that of simple fluids by assuming that the stress is more sensitive to the recent deformation than to the deformation that occurred in the distant past.

The expression for $A_{(1) i j}$ and $A_{(2) i j}$ is given by

$$
\begin{gathered}
A_{(1) i j}=v_{i, j}+v_{j, i}, \\
A_{(2) i j}=a_{i, j}+a_{j, i}+2 v^{m},{ }_{i} v_{m, j},
\end{gathered}
$$

where $v_{i}$ and $a_{i}$ are the $i$ th component of the velocity and acceleration vectors, respectively, and a comma denotes covariant differentiation with respect to the symbol following it.

\section{Basic Equations}

A rectangular Cartesian co-ordinate system is introduced such that the plate lies in $x z$-plane, $x$-axis being vertically upwards in the direction of buoyancy force which arises out of a difference in temperature of the plate and the free stream. The $y$-axis is taken perpendicular to the plate and directed into the fluid which is flowing laminarly with free stream velocity $U$. The transverse sinusoidal suction velocity distribution is assumed to be of the form

$$
\bar{v}_{w}(\bar{z})=-v_{0}\left(1+\varepsilon \cos \frac{\pi \bar{z}}{L}\right),
$$

where $\varepsilon \ll 1$ and $L$ is the wave length of the periodic suction. All physical quantities are independent of $\bar{x}$ for this problem of fully developed laminar flow.

The boundary conditions relevant to the problem are

$$
\begin{gathered}
\bar{y}=0: \bar{u}=\bar{V}, \quad \bar{v}=\bar{v}_{w}, \\
\bar{w}=0, \quad \bar{T}=\bar{T}_{w}, \quad \bar{C}=\bar{C}_{w}, \\
\bar{y} \longrightarrow \infty: \bar{u} \longrightarrow \bar{U}, \quad \bar{v} \longrightarrow-v_{0}, \quad \bar{w} \longrightarrow 0, \\
\bar{T} \longrightarrow \bar{T}_{\infty}, \quad \bar{p} \longrightarrow \bar{p}_{\infty}, \quad \bar{C} \longrightarrow \bar{C}_{\infty} .
\end{gathered}
$$

We introduce the following nondimensional quantities:

$$
\begin{aligned}
& y=\frac{\bar{y}}{L}, \quad z=\frac{\bar{z}}{L}, \quad u=\frac{\bar{u}}{v_{0}}, \\
& v=\frac{\bar{v}}{v_{0}}, \quad w=\frac{\bar{w}}{v_{0}}, \quad U=\frac{\bar{U}}{v_{0}}, \\
& \theta=\frac{\bar{T}-\bar{T}_{\infty}}{T_{w}-T_{\infty}}, \quad C=\frac{\bar{C}-\bar{C}_{\infty}}{\bar{C}_{w}-\bar{C}_{\infty}}, \quad V=\frac{\bar{V}}{v_{0}}, \\
& \operatorname{Pr}=\frac{v_{1}}{\alpha}, \quad \mathrm{S}=\frac{v_{1}}{D}, \quad p=\frac{\bar{p}}{\rho\left(v_{1} / L\right)^{2}}, \\
& p_{\infty}=\frac{\bar{p}_{\infty}}{\rho\left(v_{1} / L\right)^{2}}, \quad \mathrm{Gr}=\frac{\operatorname{Lg} \beta\left(\bar{T}_{w}-\bar{T}_{\infty}\right)}{v_{0}^{2}}, \\
& \mathrm{Gm}=\frac{\operatorname{Lg} \bar{\beta}\left(\bar{T}_{w}-\bar{T}_{\infty}\right)}{v_{0}^{2}}, \quad \operatorname{Re}=\frac{v_{0} L}{v_{1}} .
\end{aligned}
$$

Here, $(\bar{u}, \bar{v}, \bar{w})$ are the velocity components along the $(\bar{x}, \bar{y}, \bar{z})$ directions, respectively, $g$ the acceleration due to gravity, $v_{i}=\left(\mu_{i} / \rho\right)(i=1,2,3), \rho$ is the density, $\beta$ is the coefficient of volume expansion for heat transfer, $\bar{\beta}$ is the co-efficient of volume expansion for mass transfer, $\bar{p}$ is the pressure, $\bar{T}$ is the fluid temperature, $\bar{C}$ is the species concentration, $\alpha$ is the thermal diffusivity, $D$ is the chemical molecular diffusivity, $v_{0}$ is the dimensionless suction velocity, Pr is the Prandtl number, $\mathrm{S}$ is the Schmidt number, Gr is the Grashof number for heat transfer, Gm is the Grashof number for mass transfer, Re is the Reynolds number, $\bar{T}_{w}$ is the fluid temperature on the wall, and $\bar{T}_{\infty}$ is the fluid temperature in the free stream.

In view of the above nondimensional quantities, the governing equations for heat and mass transfer flow are

$$
\frac{\partial v}{\partial y}+\frac{\partial w}{\partial z}=0
$$

$$
\begin{aligned}
v \frac{\partial u}{\partial y}+ & w \frac{\partial u}{\partial z} \\
=\operatorname{Gr} \theta & +\operatorname{Gm} C+\frac{1}{\operatorname{Re}\left(\frac{\partial^{2} u}{\partial y^{2}}+\frac{\partial^{2} u}{\partial z^{2}}\right)} \\
+\alpha_{1}\left(2 \frac{\partial v}{\partial y} \frac{\partial^{2} u}{\partial y^{2}}+v \frac{\partial^{3} u}{\partial y^{3}}+\frac{\partial^{2} v}{\partial y^{2}} \frac{\partial u}{\partial y}\right. & \\
+ & \frac{\partial^{2} w}{\partial y^{2}} \frac{\partial u}{\partial z}+2 \frac{\partial w}{\partial y} \frac{\partial^{2} u}{\partial y \partial z}+w \frac{\partial^{3} u}{\partial y^{2} \partial z} \\
& +\frac{\partial^{2} v}{\partial z^{2}} \frac{\partial u}{\partial y}+2 \frac{\partial v}{\partial z} \frac{\partial^{2} u}{\partial y \partial z}+v \frac{\partial^{3} u}{\partial y \partial z^{2}} \\
& \left.+\frac{\partial^{2} w}{\partial z^{2}} \frac{\partial u}{\partial z}+2 \frac{\partial w}{\partial z} \frac{\partial^{2} u}{\partial z^{2}}+w \frac{\partial^{3} u}{\partial z^{3}}\right) \\
+\alpha_{2}( & \frac{\partial^{2} v}{\partial y^{2}} \frac{\partial u}{\partial y}+2 \frac{\partial v}{\partial y} \frac{\partial^{2} u}{\partial y^{2}}+2 \frac{\partial^{2} u}{\partial y \partial z} \frac{\partial w}{\partial y}
\end{aligned}
$$




$$
\begin{aligned}
& +\frac{\partial u}{\partial z} \frac{\partial^{2} w}{\partial y^{2}}+2 \frac{\partial^{2} u}{\partial y \partial z} \frac{\partial v}{\partial z} \\
& \left.+\frac{\partial u}{\partial z} \frac{\partial^{2} w}{\partial z^{2}}+\frac{\partial u}{\partial y} \frac{\partial^{2} v}{\partial z^{2}}+2 \frac{\partial^{2} u}{\partial z^{2}} \frac{\partial w}{\partial z}\right) \\
& v \frac{\partial v}{\partial y}+w \frac{\partial v}{\partial z} \\
& =-\frac{1}{\operatorname{Re}^{2}} \frac{\partial p}{\partial y}+\frac{1}{\operatorname{Re}}\left(\frac{\partial^{2} v}{\partial y^{2}}+\frac{\partial^{2} v}{\partial z^{2}}\right) \\
& +\alpha_{1}\left(v \frac{\partial^{3} v}{\partial y^{3}}+w \frac{\partial^{3} v}{\partial y^{2} \partial z}+v \frac{\partial^{3} v}{\partial y \partial z^{2}}\right. \\
& +w \frac{\partial^{3} v}{\partial z^{3}}+2 \frac{\partial w}{\partial y} \frac{\partial^{2} v}{\partial y \partial z}+3 \frac{\partial v}{\partial z} \frac{\partial^{2} v}{\partial y \partial z} \\
& +2 \frac{\partial^{2} u}{\partial y \partial z} \frac{\partial u}{\partial z}+13 \frac{\partial v}{\partial y} \frac{\partial^{2} v}{\partial y^{2}} \\
& +3 \frac{\partial^{2} w}{\partial y^{2}} \frac{\partial v}{\partial z}+4 \frac{\partial u}{\partial y} \frac{\partial^{2} u}{\partial y^{2}} \\
& \left.+4 \frac{\partial w}{\partial y} \frac{\partial^{2} w}{\partial y^{2}}+2 \frac{\partial u}{\partial y} \frac{\partial^{2} u}{\partial z^{2}}+\frac{\partial v}{\partial y} \frac{\partial^{2} v}{\partial z^{2}}\right) \\
& +\alpha_{2}\left(2 \frac{\partial u}{\partial y} \frac{\partial^{2} u}{\partial y^{2}}+8 \frac{\partial v}{\partial y} \frac{\partial^{2} v}{\partial y^{2}}\right. \\
& +2 \frac{\partial^{2} v}{\partial y \partial z} \frac{\partial w}{\partial y}+2 \frac{\partial^{2} w}{\partial y^{2}} \frac{\partial v}{\partial z}+2 \frac{\partial v}{\partial z} \frac{\partial^{2} v}{\partial y \partial z} \\
& \left.+2 \frac{\partial w}{\partial y} \frac{\partial^{2} w}{\partial y^{2}}+\frac{\partial^{2} u}{\partial y \partial z} \frac{\partial u}{\partial z}+\frac{\partial u}{\partial y} \frac{\partial^{2} u}{\partial z^{2}}\right), \\
& v \frac{\partial w}{\partial y}+w \frac{\partial w}{\partial z} \\
& =-\frac{1}{\operatorname{Re}^{2}} \frac{\partial p}{\partial z}+\frac{1}{\operatorname{Re}}\left(\frac{\partial^{2} w}{\partial y^{2}}+\frac{\partial^{2} w}{\partial z^{2}}\right) \\
& +\alpha_{1}\left(w \frac{\partial^{3} v}{\partial y^{3}}+v \frac{\partial^{3} w}{\partial y \partial z^{2}}+w \frac{\partial^{3} w}{\partial z^{3}}\right. \\
& +v \frac{\partial^{3} w}{\partial y^{3}}+2 \frac{\partial v}{\partial z} \frac{\partial^{2} w}{\partial y \partial z}+3 \frac{\partial w}{\partial y} \frac{\partial^{2} w}{\partial y \partial z} \\
& +2 \frac{\partial u}{\partial y} \frac{\partial^{2} u}{\partial y \partial z}+13 \frac{\partial w}{\partial z} \frac{\partial^{2} w}{\partial z^{2}} \\
& +3 \frac{\partial^{2} v}{\partial z^{2}} \frac{\partial w}{\partial y}+4 \frac{\partial u}{\partial z} \frac{\partial^{2} u}{\partial z^{2}} \\
& \left.+4 \frac{\partial v}{\partial z} \frac{\partial^{2} v}{\partial z^{2}}+2 \frac{\partial u}{\partial z} \frac{\partial^{2} u}{\partial y^{2}}+\frac{\partial w}{\partial z} \frac{\partial^{2} w}{\partial y^{2}}\right)
\end{aligned}
$$

$$
\begin{gathered}
+\alpha_{2}\left(2 \frac{\partial u}{\partial z} \frac{\partial^{2} u}{\partial z^{2}}+8 \frac{\partial w}{\partial z} \frac{\partial^{2} w}{\partial z^{2}}+2 \frac{\partial v}{\partial z} \frac{\partial^{2} w}{\partial y \partial z}\right. \\
+2 \frac{\partial w}{\partial y} \frac{\partial^{2} v}{\partial z^{2}}+2 \frac{\partial w}{\partial y} \frac{\partial^{2} w}{\partial y \partial z} \\
\left.+2 \frac{\partial v}{\partial z} \frac{\partial^{2} v}{\partial z^{2}}+\frac{\partial^{2} u}{\partial y \partial z} \frac{\partial u}{\partial y}+\frac{\partial u}{\partial z} \frac{\partial^{2} u}{\partial y^{2}}\right) \\
v \frac{\partial \theta}{\partial y}+w \frac{\partial \theta}{\partial z}=\frac{1}{\operatorname{Re} \operatorname{Pr}}\left(\frac{\partial^{2} \theta}{\partial y^{2}}+\frac{\partial^{2} \theta}{\partial z^{2}}\right) \\
v \frac{\partial C}{\partial y}+w \frac{\partial C}{\partial z}=\frac{1}{\operatorname{Re~S}}\left(\frac{\partial^{2} C}{\partial y^{2}}+\frac{\partial^{2} C}{\partial z^{2}}\right)
\end{gathered}
$$

where $\alpha_{1}=v_{2} / L^{2}$ and $\alpha_{2}=v_{3} / L^{2}$.

The corresponding boundary conditions are

$$
\begin{array}{r}
y=0: u=V, \quad v(z)=-(1+\varepsilon \cos \pi z), \\
w=0, \quad \theta=1, \quad C=1, \\
y \longrightarrow \infty: u \longrightarrow U, \quad v \longrightarrow-1, \\
w \longrightarrow 0, \theta \longrightarrow 0, \quad C \longrightarrow 0, \quad p \longrightarrow p_{\infty} .
\end{array}
$$

\section{Method of Solution}

When the amplitude $\varepsilon(\ll 1)$ of the suction velocity is small, we assume the solutions of the nonlinear partial differential equations (7) to (11) of the form

$$
\begin{aligned}
& u(y, z)=u_{0}(y)+\varepsilon u_{1}(y, z)+o\left(\varepsilon^{2}\right), \\
& v(y, z)=v_{0}(y)+\varepsilon v_{1}(y, z)+o\left(\varepsilon^{2}\right), \\
& w(y, z)=w_{0}(y)+\varepsilon w_{1}(y, z)+o\left(\varepsilon^{2}\right), \\
& p(y, z)=p_{0}(y)+\varepsilon p_{1}(y, z)+o\left(\varepsilon^{2}\right), \\
& \theta(y, z)=\theta_{0}(y)+\varepsilon \theta_{1}(y, z)+o\left(\varepsilon^{2}\right), \\
& C(y, z)=C_{0}(y)+\varepsilon C_{1}(y, z)+o\left(\varepsilon^{2}\right) .
\end{aligned}
$$

When $\varepsilon=0$, the problem reduces to the two-dimensional case with $w=w_{0}=0$ and $p=p_{0}=p_{\infty}$. Substituting (13) into (6) to (11), the terms free from $\varepsilon$ of both sides are

$$
\begin{aligned}
& v_{0}^{\prime}=0 \text {, } \\
& \alpha_{1} v_{0} u_{0}^{\prime \prime}+\frac{1}{\operatorname{Re}} u_{0}^{\prime \prime}-v_{0} u_{0}^{\prime}+\mathrm{Gr} \theta_{0}+\mathrm{Gm} C_{0}=0 \text {, } \\
& -\frac{1}{\operatorname{Re}^{2}} \frac{\partial p_{0}}{\partial y}+\left(4 \alpha_{1}+2 \alpha_{2}\right) \frac{\partial u_{0}}{\partial y} \frac{\partial^{2} u_{0}}{\partial y^{2}}=0, \\
& -\frac{\partial \theta_{0}}{\partial y}=\frac{1}{\operatorname{Re} \operatorname{Pr}} \frac{\partial^{2} \theta_{0}}{\partial y^{2}} \\
& -\frac{\partial C_{0}}{\partial y}=\frac{1}{\operatorname{ReS}} \frac{\partial^{2} C_{0}}{\partial y^{2}}
\end{aligned}
$$

where prime denotes differentiation with respect to $y$. 
The corresponding boundary conditions are

$$
\begin{gathered}
y=0: u_{0}=V, \quad v_{0}=-1, \\
w_{0}=0, \quad \theta_{0}=1, \quad C_{0}=1, \\
y \longrightarrow \infty: u_{0} \longrightarrow U, \quad v_{0} \longrightarrow-1, \quad w_{0} \longrightarrow 0, \\
\theta_{0} \longrightarrow 0, \quad C_{0} \longrightarrow 0, \quad p_{0} \longrightarrow p_{\infty} .
\end{gathered}
$$

Solving (14), (17), and (18) under boundary conditions (19), we get

$$
\begin{aligned}
& v_{0}=-1, \\
& \theta_{0}=\exp (-\operatorname{Re} \operatorname{Pr} y), \\
& C_{0}=\exp (-\operatorname{Re} \operatorname{S} y) .
\end{aligned}
$$

As $\left|\alpha_{1}\right| \ll 1$ (due to small shear rate) therefore substituting

$$
u_{0}(y)=u_{00}(y)+\alpha_{1} u_{01}(y)+o\left(\alpha_{1}^{2}\right),
$$

into (15) and boundary conditions (19) up to the first order of $\alpha_{1}$, and equating the coefficients of like powers of $\alpha_{1}$, we obtain the following sets of ordinary differential equations and corresponding boundary conditions

$$
\begin{aligned}
u_{00}^{\prime \prime}+\operatorname{Re} u_{00}^{\prime}+ & \operatorname{Re}\{\operatorname{Grexp}(-\operatorname{Re} \operatorname{Pr} y) \\
+ & \operatorname{Gm} \exp (-\operatorname{Re} \mathrm{S} y)\}=0,
\end{aligned}
$$

$$
\operatorname{Re} u_{00}^{\prime \prime \prime}-u_{01}^{\prime \prime}-\operatorname{Re} u_{01}^{\prime}=0 \text {, }
$$

$$
\begin{aligned}
& y=0: u_{00}=V, \quad u_{01}=0, \\
& y \longrightarrow \infty: u_{00} \longrightarrow U, \quad u_{01} \longrightarrow 0 .
\end{aligned}
$$

Solving (22) and (23) under boundary conditions (24) and then substituting into (21) (neglecting $\alpha_{1}^{2}$ ), we get

$$
\begin{aligned}
u_{0}= & U+(V-U) \exp (-\operatorname{Re} y) \\
+ & \frac{\mathrm{Gr}}{\operatorname{Re} \operatorname{Pr}(\operatorname{Pr}-1)}\{\exp (-\operatorname{Re} y)-\exp (-\operatorname{Re} \operatorname{Pr} y)\} \\
+ & \frac{\mathrm{Gm}}{\operatorname{Re} \mathrm{S}(\mathrm{S}-1)}\{\exp (-\operatorname{Re} y)-\exp (-\operatorname{Re} \mathrm{S} y)\} \\
- & \frac{\alpha_{1}}{\operatorname{Re}}\left[\left\{\frac{A_{2}}{\operatorname{Pr}(\operatorname{Pr}-1)}+\frac{A_{3}}{\mathrm{~S}(\mathrm{~S}-1)}\right\} \exp (-\operatorname{Re} y)\right. \\
& +A_{1} \operatorname{Re} y \exp (-\operatorname{Re} y) \\
& \left.\quad \frac{A_{2} \exp (-\operatorname{Re} \operatorname{Pr} y)}{\operatorname{Pr}(\operatorname{Pr}-1)}-\frac{A_{3} \exp (-\operatorname{Re} \mathrm{S} y)}{\mathrm{S}(\mathrm{S}-1)}\right],
\end{aligned}
$$

where $A_{1}=\operatorname{Re}^{3}\{U-V-(\mathrm{Gr} / \operatorname{Pr} \operatorname{Re}(\operatorname{Pr}-1))-(\mathrm{Gm} / \mathrm{Re} \mathrm{S}(\mathrm{S}-$ 1)) $\}, A_{2}=\left(\mathrm{GrRe}^{2} \operatorname{Pr}^{2} /(\mathrm{Pr}-1)\right), A_{3}=\mathrm{Gm} \mathrm{Re}^{2} \mathrm{~S}^{2} /(\mathrm{S}-$ 1) with $\operatorname{Pr} \neq 1, S \neq 1$.

Equating the coefficients of $\varepsilon$ from both sides after substitution of (13) in (6) to (11), neglecting those of $\varepsilon^{2}$, using (16), and assuming $\partial p_{0} / \partial y=0$, we get

$$
\frac{\partial v_{1}}{\partial u}+\frac{\partial w_{1}}{\partial z}=0
$$

$$
\begin{aligned}
v_{1} \frac{\partial u_{0}}{\partial y}-\frac{\partial u_{1}}{\partial y}= & \operatorname{Gr} \theta_{1}+\operatorname{Gm} C_{1}+\frac{1}{\operatorname{Re}}\left(\frac{\partial^{2} u_{1}}{\partial y^{2}}+\frac{\partial^{2} u_{1}}{\partial z^{2}}\right) \\
+\alpha_{1} & \left(-2 \frac{\partial v_{1}}{\partial y} \frac{\partial^{2} u_{0}}{\partial y^{2}}-\frac{\partial^{3} u_{1}}{\partial y^{3}}+v_{1} \frac{\partial^{3} u_{0}}{\partial y^{3}}\right. \\
& \left.-\frac{\partial^{2} v_{1}}{\partial y^{2}} \frac{\partial u_{0}}{\partial y}-\frac{\partial u_{0}}{\partial y} \frac{\partial^{2} v_{1}}{\partial z^{2}}-\frac{\partial^{3} u_{1}}{\partial y \partial z^{2}}\right),
\end{aligned}
$$

$$
\begin{aligned}
-\frac{\partial v_{1}}{\partial y}= & -\frac{1}{\operatorname{Re}^{2}} \frac{\partial p_{1}}{\partial y}+\frac{1}{\operatorname{Re}}\left(\frac{\partial^{2} v_{1}}{\partial y^{2}}+\frac{\partial^{2} v_{1}}{\partial z^{2}}\right) \\
& -\alpha_{1}\left(\frac{\partial^{3} v_{1}}{\partial y^{3}}+\frac{\partial^{3} v_{1}}{\partial y \partial z^{2}}\right),
\end{aligned}
$$

$$
\begin{gathered}
-\frac{\partial w_{1}}{\partial y}=-\frac{1}{\operatorname{Re}^{2}} \frac{\partial p_{1}}{\partial z}+\frac{1}{\operatorname{Re}}\left(\frac{\partial^{2} w_{1}}{\partial y^{2}}+\frac{\partial^{2} w_{1}}{\partial z^{2}}\right) \\
-\alpha_{1}\left(\frac{\partial^{3} w_{1}}{\partial y \partial z^{2}}+\frac{\partial^{3} w_{1}}{\partial y^{3}}\right), \\
-\frac{\partial \theta_{1}}{\partial y}+v_{1} \frac{\partial \theta_{0}}{\partial y}=\frac{1}{\operatorname{Re} \operatorname{Pr}}\left(\frac{\partial^{2} \theta_{1}}{\partial y^{2}}+\frac{\partial^{2} \theta_{1}}{\partial z^{2}}\right), \\
-\frac{\partial C_{1}}{\partial y}+v_{1} \frac{\partial C_{0}}{\partial y}=\frac{1}{\operatorname{Re~S}}\left(\frac{\partial^{2} C_{1}}{\partial y^{2}}+\frac{\partial^{2} C_{1}}{\partial z^{2}}\right),
\end{gathered}
$$

with relevant boundary conditions

$$
\begin{aligned}
& y=0: \quad u_{1}=0, \quad v_{1}=-\cos \pi z, \\
& w_{1}=0, \quad \theta_{1}=0, \quad p_{1}=0, \quad C_{1}=0, \\
& y \longrightarrow \infty: \quad u_{1} \longrightarrow 0, \quad v_{1} \longrightarrow 0, \quad w_{1} \longrightarrow 0, \\
& \theta_{1} \longrightarrow 0, \quad p_{1} \longrightarrow 0, \quad C_{1} \longrightarrow 0 .
\end{aligned}
$$

Equations (26), (28), and (29) govern the cross-flow, and (27), (30), and (31) govern the main flow, the temperature and the species concentration, respectively.

\section{Cross-Flow Solution}

In order to solve (26), (28), and (29), being independent of the main flow component $u_{1}$ and the temperature field $\theta_{1}$, we assume that

$$
\begin{gathered}
v_{1}(y, z)=-\pi v_{11}(y) \cos \pi z, \\
w_{1}(y, z)=v_{11}^{\prime}(y) \sin \pi z, \\
p_{1}(y, z)=\operatorname{Re}^{2} p_{11}(y) \cos \pi z .
\end{gathered}
$$

The prime in $v_{11}^{\prime}$ denotes differentiation with respect to $y$. Equations (33) and (34) have been chosen so that the continuity equation (26) is satisfied. Substituting these equations into (28) and (29), two ordinary differential 
equations for $v_{11}$ and $p_{11}$ are obtained:

$$
\begin{aligned}
& v_{11}^{\prime \prime}+\operatorname{Re} v_{11}^{\prime}-\pi^{2} v_{11}+\alpha_{1} \operatorname{Re}\left(\pi^{2} v_{11}^{\prime}-v_{11}^{\prime \prime \prime}\right)=-\frac{p_{11}^{\prime}}{\pi} \operatorname{Re} \\
& v_{11}^{\prime \prime \prime}+\operatorname{Re} v_{11}^{\prime \prime}-\pi^{2} v_{11}^{\prime}+\alpha_{1} \operatorname{Re}\left(\pi^{2} v_{11}^{\prime \prime}-v_{11}^{\mathrm{IV}}\right)=-\pi p_{11} \operatorname{Re}
\end{aligned}
$$

with the boundary conditions

$$
\begin{aligned}
& y=0: \quad v_{11}=\frac{1}{\pi}, \quad v_{11}^{\prime}=0, \\
& y \longrightarrow 0: v_{11} \longrightarrow 0, \quad v_{11}^{\prime} \longrightarrow 0 .
\end{aligned}
$$

On eliminating the pressure $p_{11}^{\prime}$ from (36) and (37), we get the following differential equation in $v_{11}$ as

$$
\begin{gathered}
v_{11}^{\mathrm{IV}}+\operatorname{Re} v_{11}^{\prime \prime \prime}-2 \pi^{2} v_{11}^{\prime}-\pi^{2} \operatorname{Re} v_{11}^{\prime}+\pi^{4} v_{11} \\
+\alpha_{1} \operatorname{Re}\left(2 \pi^{2} v_{11}^{\prime \prime \prime}-v_{11}^{\mathrm{V}}-\pi^{4} v_{11}^{\prime}\right)=0
\end{gathered}
$$

To solve (39), we note that $\alpha_{1}<1$ for small shear, and so we can assume that

$$
v_{11}(y)=v_{110}(y)+\alpha_{1} v_{111}(y)+o\left(\alpha_{1}^{2}\right) .
$$

Substituting from (40) into (39) then equating the like powers of $\alpha_{1}$ and neglecting the higher powers of $\alpha_{1}$, we get

$$
\begin{aligned}
& v_{110}^{\mathrm{IV}}+\operatorname{Re} v_{110}^{\prime \prime \prime}-2 \pi^{2} v_{110}^{\prime \prime}-\pi^{2} \operatorname{Re} v_{110}^{\prime}+\pi^{4} v_{110}=0, \\
& v_{111}^{\mathrm{IV}}+\operatorname{Re} v_{111}^{\prime \prime \prime}-2 \pi^{2} v_{111}^{\prime \prime}-\pi^{2} \operatorname{Re} v_{111}^{\prime}+\pi^{4} v_{111}+2 \operatorname{Re} \pi^{2} v_{110}^{\prime \prime \prime} \\
& \quad-\operatorname{Re} v_{110}^{\mathrm{V}}-\pi^{4} \operatorname{Re} v_{110}^{\prime}=0 .
\end{aligned}
$$

The corresponding boundary conditions are:

$$
\begin{aligned}
& y=0: \quad v_{110}=\frac{1}{\pi}, \quad v_{111}=0, \\
& v_{110}^{\prime}=0, \quad v_{111}^{\prime}=0, \\
& y \longrightarrow \infty: \quad v_{110} \longrightarrow 0, \quad v_{111} \longrightarrow 0, \\
& v_{110}^{\prime} \longrightarrow 0, \quad v_{111}^{\prime} \longrightarrow 0 .
\end{aligned}
$$

Solving (41) and (42) under boundary conditions (43), we get the expression for $v_{11}$ and hence the solutions for velocity components $v_{1}, w_{1}$ and the pressure $p_{1}$.

\section{Solution for Main Flow, Temperature, and Molar Concentration Fields}

To solve (27), (30), and (31), we assume the following form:

$$
\begin{aligned}
& u_{1}(y, z)=u_{11}(y) \cos \pi z, \\
& \theta_{1}(y, z)=\theta_{11}(y) \cos \pi z \\
& C_{1}(y, z)=C_{11}(y) \cos \pi z .
\end{aligned}
$$

On using (44) into (27), (30), and (31), we get

$$
\begin{gathered}
u_{11}^{\prime \prime}-\pi^{2} u_{11}+\operatorname{Re}\left(\pi v_{11} u_{0}^{\prime}+u_{11}^{\prime}\right)+\operatorname{Gr} \theta_{11}+\operatorname{Gm} C_{11} \\
+\alpha_{1}\left(2 \pi v_{11}^{\prime} u_{0}^{\prime \prime}-u_{11}^{\prime \prime \prime}-\pi v_{11} u_{0}^{\prime \prime \prime}+\pi v_{11}^{\prime \prime} u_{0}^{\prime}\right. \\
\left.-u_{0}^{\prime} \pi^{3} v_{11}+\pi^{2} u_{11}^{\prime}\right)=0 \\
\theta_{11}^{\prime \prime}-\pi^{2} \theta_{11}+\operatorname{Re} \operatorname{Pr}\left(\theta_{11}^{\prime}+\pi v_{11} \theta_{0}^{\prime}\right)=0, \\
C_{11}^{\prime \prime}-\pi^{2} C_{11}+\operatorname{Re} \mathrm{S}\left(C_{11}^{\prime}+\pi v_{11} C_{0}^{\prime}\right)=0,
\end{gathered}
$$

subject to boundary conditions

$$
\begin{aligned}
& y=0: u_{11}=0, \quad \theta_{11}=0, \quad C_{11}=0 \\
& y \longrightarrow \infty: u_{11} \longrightarrow 0 \\
& \theta_{11} \longrightarrow 0, \quad C_{11} \longrightarrow 0
\end{aligned}
$$

Solving (46) and (47) under boundary conditions (48), we get the expressions for $\theta_{11}$ and $C_{11}$, and hence the solutions for $\theta_{1}$ and $C_{1}$ have been obtained.

Again, substituting

$$
u_{11}(y)=u_{110}(y)+\alpha_{1} u_{111}(y)+o\left(\alpha_{1}^{2}\right)
$$

into (45) and boundary conditions (48) up to first order of $\alpha_{1}$ and comparing the coefficients of like powers of $\alpha_{1}$, we obtain

$$
\begin{array}{r}
u_{110}^{\prime \prime}+\operatorname{Re} u_{110}^{\prime}-\pi^{2} u_{110}=-\operatorname{Re}\left(\operatorname{Gr} \theta_{11}+\operatorname{Gm} C_{11}+\pi v_{11} u_{0}^{\prime}\right), \\
u_{111}^{\prime \prime}+\operatorname{Re} u_{111}^{\prime}-\pi^{2} u_{111}=\operatorname{Re}\left(-2 \pi v_{11}^{\prime} u_{0}^{\prime \prime}+u_{110}^{\prime \prime \prime}+\pi v_{11} u_{0}^{\prime \prime \prime},\right. \\
\left.-\pi v_{11}^{\prime \prime} u_{0}^{\prime}+u_{0}^{\prime} \pi^{3} v_{11}-\pi^{2} u_{110}^{\prime}\right),
\end{array}
$$

subject to boundary conditions

$$
\begin{aligned}
& y=0: u_{110}=0, \quad u_{111}=0, \\
& y \longrightarrow \infty: u_{110} \longrightarrow 0, \quad u_{111} \longrightarrow 0 .
\end{aligned}
$$

Solving (50) and (51) under boundary conditions (52), we get the expression for $u_{110}$ and $u_{111}$, and hence the solutions for $u_{11}$ and $u_{1}$ have been obtained. The solutions of the differential equations are not presented here for the sake of brevity.

\section{Results and Discussion}

The nondimensional skin friction coefficient $\sigma_{x y}$ at the plate $y=0$ in the main flow direction is

$$
\begin{aligned}
\sigma_{x y}= & \frac{\bar{\sigma}_{x y}}{\rho v_{0}^{2}}=\left(\frac{1}{\operatorname{Re}} \frac{\partial u}{\partial y}\right)_{y=0}+\alpha_{1} \\
& \times\left(v \frac{\partial^{2} u}{\partial y^{2}}+w \frac{\partial^{2} u}{\partial y \partial z}-3 \frac{\partial u}{\partial y} \frac{\partial v}{\partial y}-\frac{\partial w}{\partial y} \frac{\partial u}{\partial z}-2 \frac{\partial u}{\partial z} \frac{\partial v}{\partial z}\right)_{y=0} .
\end{aligned}
$$


TABLE 1: Various combinations of flow parameters.

\begin{tabular}{lcccc}
\hline Cases & $\mathrm{Gm}$ & $\mathrm{Pr}$ & $\mathrm{Re}$ & $\mathrm{S}$ \\
\hline $\mathrm{I}$ & 2 & 3 & 3.5 & 0.1 \\
II & 5 & 3 & 3.5 & 0.1 \\
III & 5 & 5 & 3.5 & 0.1 \\
IV & 5 & 5 & 4.5 & 0.1 \\
V & 5 & 5 & 4.5 & 0.6 \\
\hline
\end{tabular}

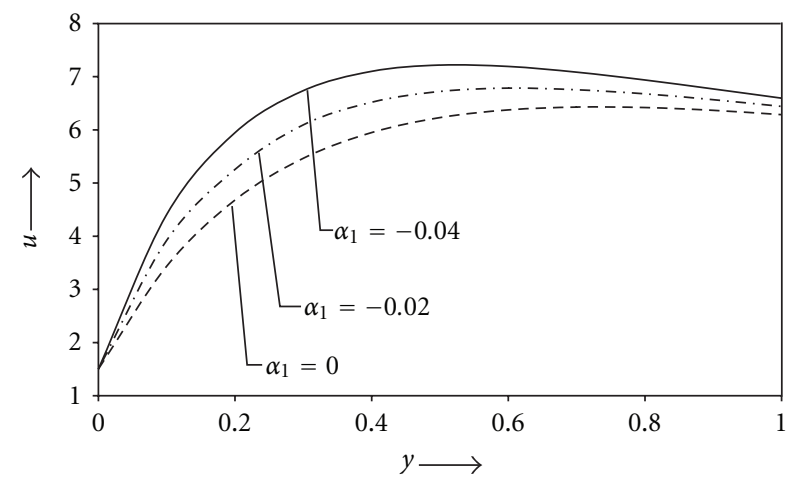

Figure 1: Variation of $u$ against $y$ for case I.

The nondimensional heat flux at the plate $y=0$ in terms of Nusselt number $\mathrm{Nu}$ is

$$
\begin{aligned}
\mathrm{Nu} & =\frac{k}{\rho v_{0} C_{p}\left(\bar{T}_{w}-\bar{T}_{\infty}\right)}\left(\frac{\partial \bar{T}}{\partial \bar{y}}\right) \\
& =\frac{1}{\operatorname{Pr} \operatorname{Re}}\left[\theta_{0}^{\prime}(0)+\varepsilon \theta_{11}^{\prime}(0) \sin \pi z\right] .
\end{aligned}
$$

The nondimensional mass flux at the plate $y=0$ in terms of Sherwood number $\mathrm{Sh}_{0}$ is

$$
\begin{aligned}
\mathrm{Sh}_{0} & =\frac{D}{v_{0}\left(\bar{C}_{w}-\bar{C}_{\infty}\right)}\left(\frac{\partial \bar{C}}{\partial \bar{y}}\right)_{y=0} \\
& =\frac{1}{\operatorname{SRe}}\left[C_{0}^{\prime}(0)+\varepsilon C_{11}^{\prime}(0) \cos \pi z\right] .
\end{aligned}
$$

The purpose of this study is to bring out the effects of the non-Newtonian parameter on the governing flow with the combinations of the other flow parameters as the effects of the other parameters discussed by Ahmed. The nonNewtonian effect is exhibited through the nondimensional visco-elastic parameter $\alpha_{1}$. The corresponding results for Newtonian fluid are obtained by setting $\alpha_{1}=0$.

Figures 1 to 5 represent the velocity profiles $u$ against $y$ to observe the visco-elastic effects for various sets of values (Table 1) of the Grashof number Gm for mass transfer, Prandtl number Pr, Reynolds number Re and Schmidt number S. It is evident from Figures 1 to 5 that the values of the velocity $u$ increase with the increasing values of the non-Newtonian parameter $\left|\alpha_{1}\right|,\left(\alpha_{1}=0,-0.02,-0.04\right)$ in comparison with the Newtonian fluid $\left(\alpha_{1}=0\right)$ for all the cases in Table 1.

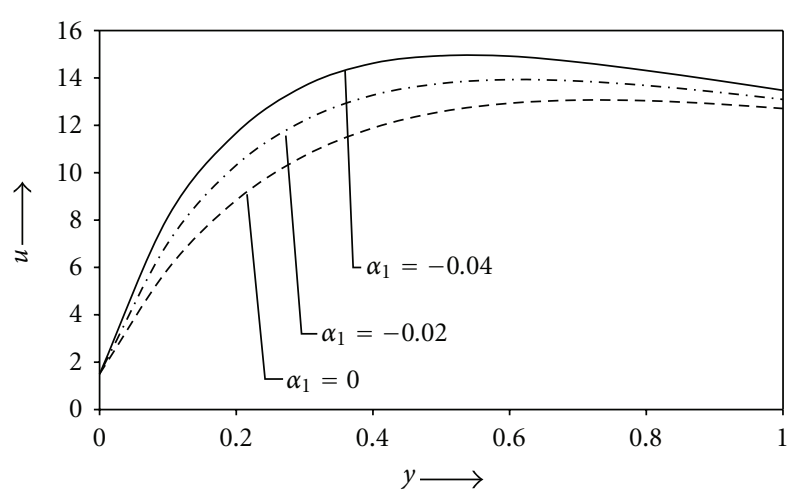

FIgURE 2: Variation of $u$ against $y$ for case II.

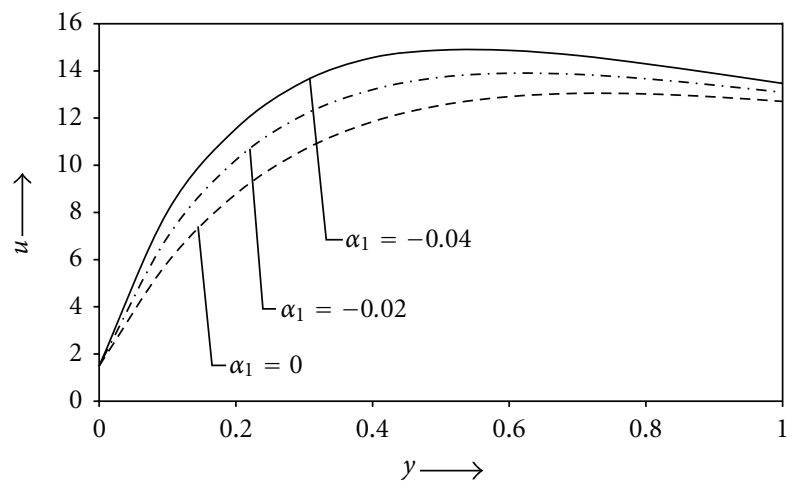

Figure 3: Variation of $u$ against $y$ for case III.

Figures 6 to 8 exhibit the effects of $\left|\alpha_{1}\right|$ on shear stress $\sigma$ against $\mathrm{Gm}$ for various sets of values of $\mathrm{S}$ and Pr. It is evident from the Figures 6 to 8 that the values of $\sigma$ decrease with the increasing values of the non-Newtonian parameter $\left|\alpha_{1}\right|$ in comparison with the Newtonian fluid for increasing values of the Schmidt number S (Figures 6 and 7) or Prandtl number $\operatorname{Pr}$ (Figures 7 and 8 ).

Figures 9 to 11 depict the shear stress $\sigma$ against Schmidt number $\mathrm{S}$ for various sets of values of the Grashof number Gm for mass transfer and Prandtl number Pr. It is observed from the figures that the shear stress decreases with the increasing values of the non-Newtonian parameter $\left|\alpha_{1}\right|$, in comparison to the Newtonian fluid for increasing values of Grashof number (Figures 9 and 10) or Prandtl number (Figures 10 and 11).

It has also been observed that the heat and mass flux at the plate $y=0$ are not significantly affected by the nonNewtonian parameter.

\section{Conclusions}

The present work is an attempt to study the viscoelastic effects on free convective three-dimensional flow along a steadily moving porous vertical plate in presence of transverse sinusoidal suction velocity and uniform free stream velocity. The second-order fluid model for a viscoelastic fluid flow is assumed. The effects of viscoelastic parameter 


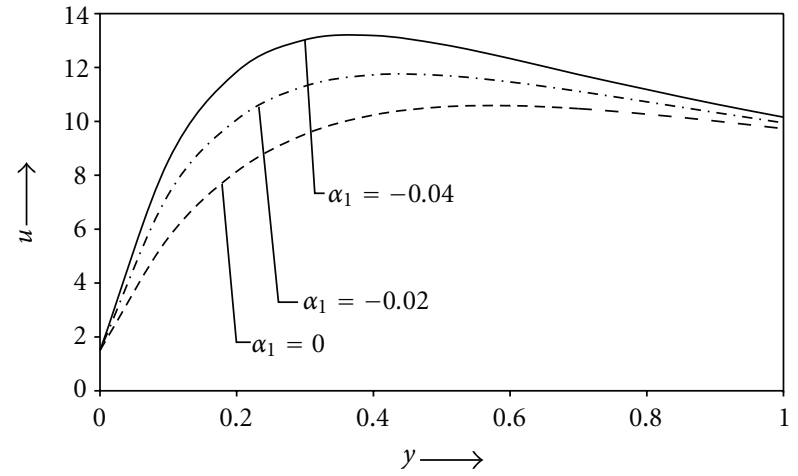

FIgURE 4: Variation of $u$ against $y$ for case IV.

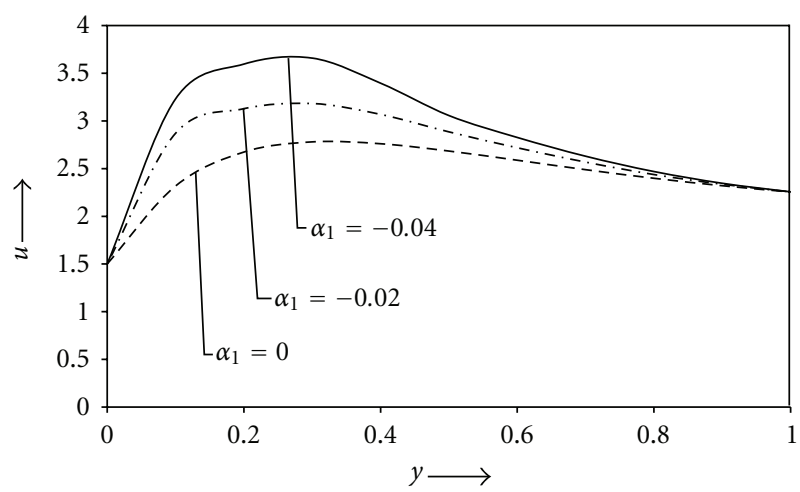

FIgURE 5: Variation of $u$ against $y$ for case V.

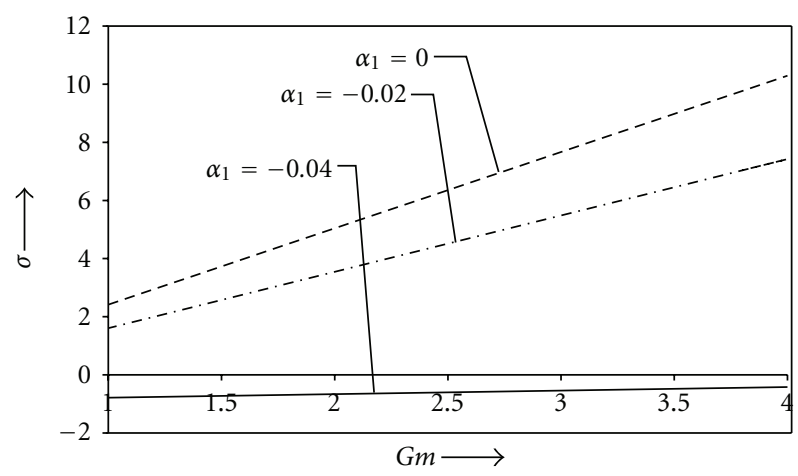

Figure 6: Variation of $\sigma$ against $\mathrm{Gm}$ for $s=0.1, \operatorname{Pr}=3$.

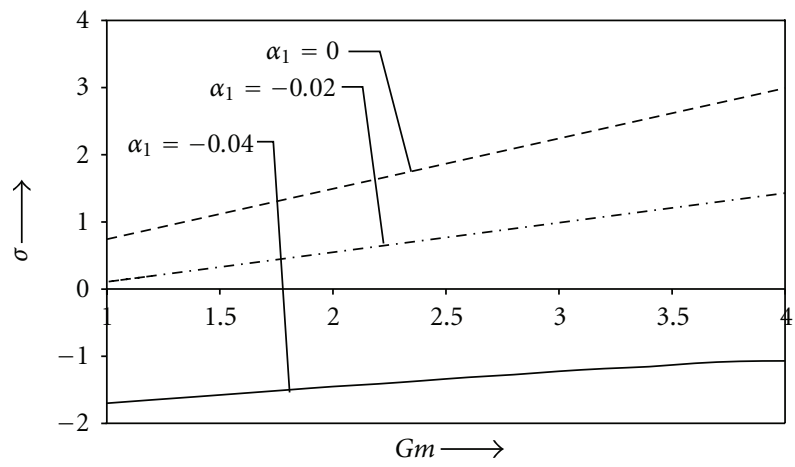

FIgURE 7: Variation of $\sigma$ against $\mathrm{Gm}$ for $s=0.4, \operatorname{Pr}=3$.

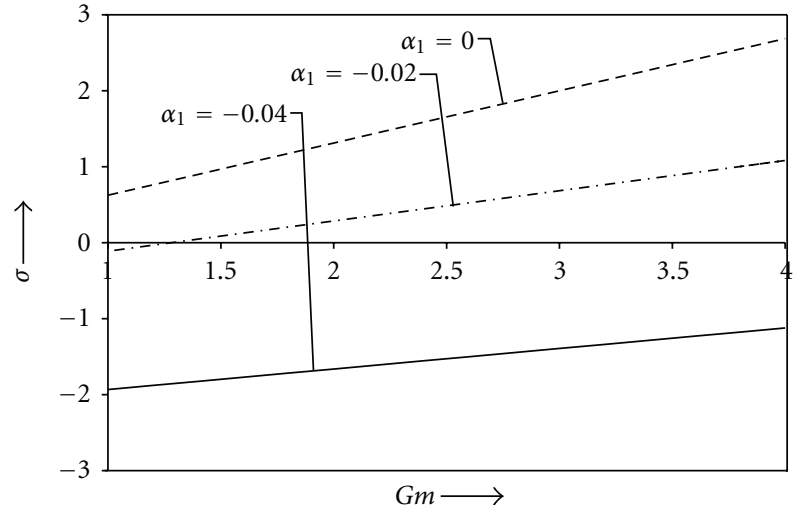

Figure 8: Variation of $\sigma$ against $\mathrm{Gm}$ for $s=0.4, \operatorname{Pr}=5$.

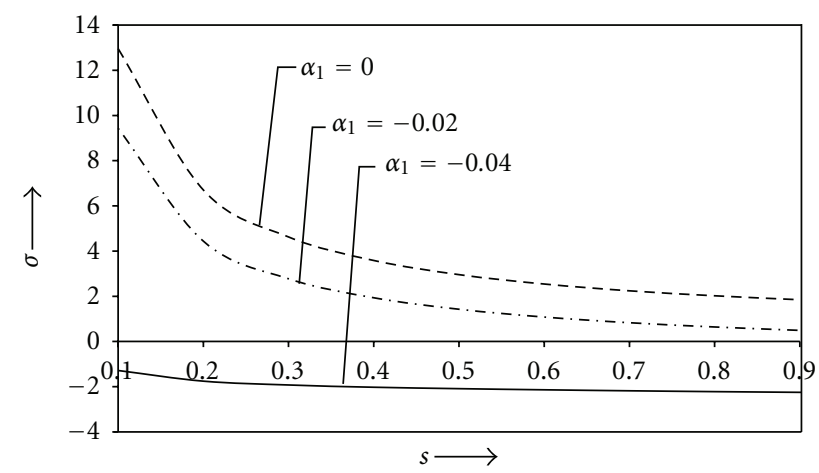

Figure 9: Variation of $\sigma$ against $s$ for $\mathrm{Gm}=2, \operatorname{Pr}=3$.

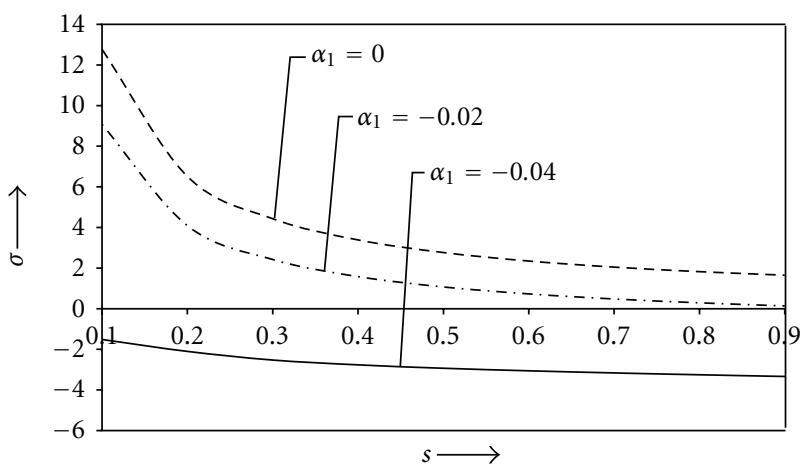

FIGURE 10: Variation of $\sigma$ against $s$ for $\mathrm{Gm}=5, \operatorname{Pr}=3$.

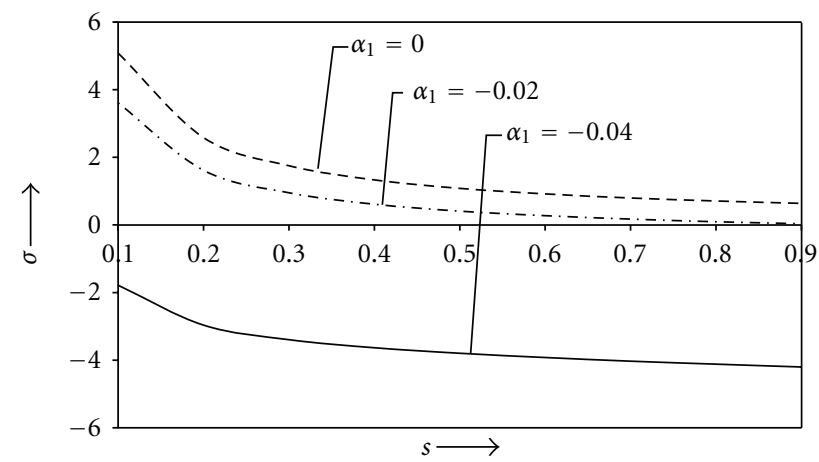

Figure 11: Variation of $\sigma$ against $s$ for $\mathrm{Gm}=5, \operatorname{Pr}=5$. 
on velocity profile for different $\mathrm{Gm}, \mathrm{Pr}, \mathrm{Re}$, and $\mathrm{S}$ are studied in detail. It was found that the velocity increases with the increasing values of the visco-elastic parameter in comparison to the Newtonian fluid. Also, in all the cases studied, it was found that the shear stress decreases with the increasing values of the viscoelastic parameter in comparison with the Newtonian fluid. Further, it was observed that the shear stress increases with the increasing values of Grashof number Gm for both Newtonian and non-Newtonian cases. But shear stress decreases with the increasing values of the Schmidt number for both Newtonian and non-Newtonian cases.

\section{References}

[1] V. M. Soundalgekar and P. D. Wavre, "Unsteady free convection flow past an infinite vertical plate with constant suction and mass transfer," International Journal of Heat and Mass Transfer, vol. 20, no. 12, pp. 1363-1373, 1977.

[2] H. T. Lin and C. M. Wu, "Combined heat and mass transfer by laminar natural convection from a vertical plate," Heat and Mass Transfer, vol. 30, no. 6, pp. 369-376, 1995.

[3] R. Muthucumaraswamy, P. Ganesan, and V. M. Soundalgekar, "Heat and mass transfer effects on flow past an impulsively started vertical plate," Acta Mechanica, vol. 146, no. 1-2, pp. 1-8, 2001.

[4] G. V. Lachman, Boundary Layer and Flow Control, Its Principles and Application, Vols. I and II, Pergamon Press, 1961.

[5] P. Singh, V. P. Sharma, and U. N. Misra, "Fluctuating boundary layer on a heated horizontal plate," Acta Mechanica, vol. 30, no. 1-2, pp. 111-128, 1978.

[6] K. D. Singh and R. Sharma, "Three dimensional Couette flow through a porous medium with heat transfer," Indian Journal of Pure and Applied Mathematics, vol. 32, no. 12, pp. 18191829, 2001.

[7] R. C. Chaudhary and P. Kumar Sharma, "Three dimensional unsteady convection and mass transfer flow through porous medium," Heat and Mass Transfer, vol. 39, no. 8-9, pp. 765770, 2003.

[8] S. Ahmed, "Free and forced convective three dimensional flow with heat and mass transfer," International Journal of Applied Mathematics and Mechanics, vol. 5, no. 1, pp. 26-38, 2009.

[9] H. Markovitz and B. D. Coleman, "Incompressible secondorder fluids," Advances in Applied Mechanics, vol. 8, pp. 69101, 1964.

[10] B. D. Coleman and W. Noll, "An approximation theorem for functionals, with applications in continuum mechanics," Archive for Rational Mechanics and Analysis, vol. 6, no. 1, pp. 355-370, 1960. 


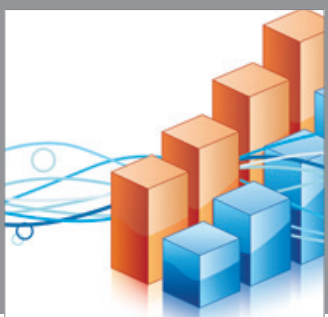

Advances in

Operations Research

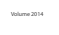

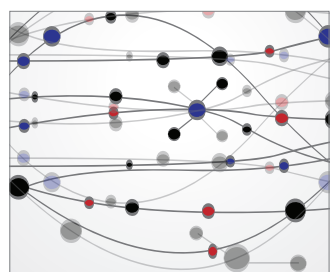

\section{The Scientific} World Journal
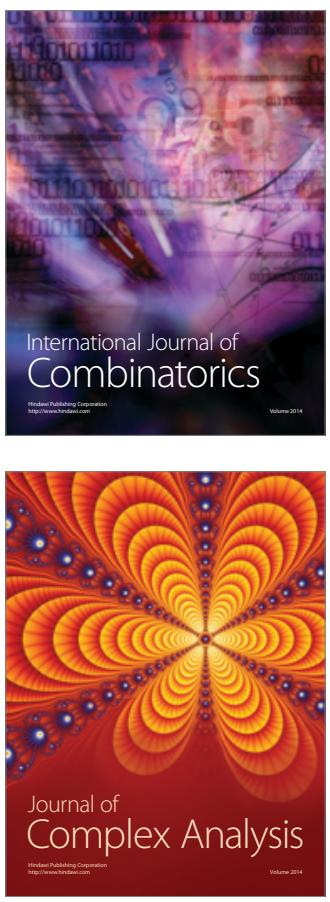

International Journal of

Mathematics and

Mathematical

Sciences
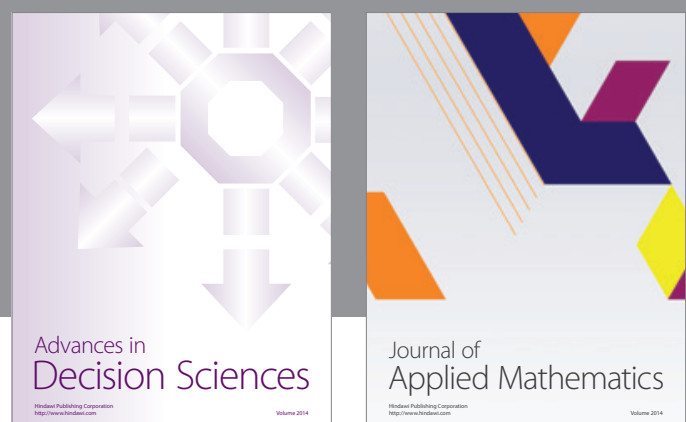

Journal of

Applied Mathematics
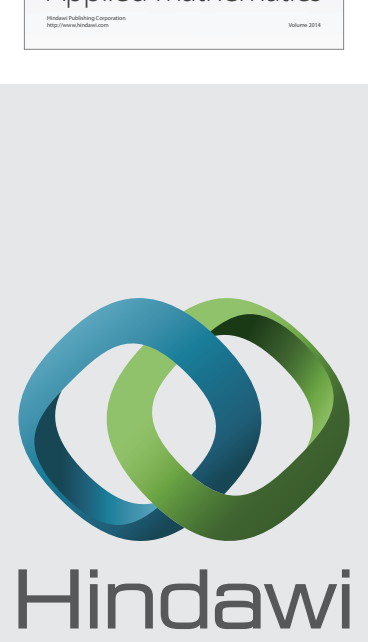

Submit your manuscripts at http://www.hindawi.com
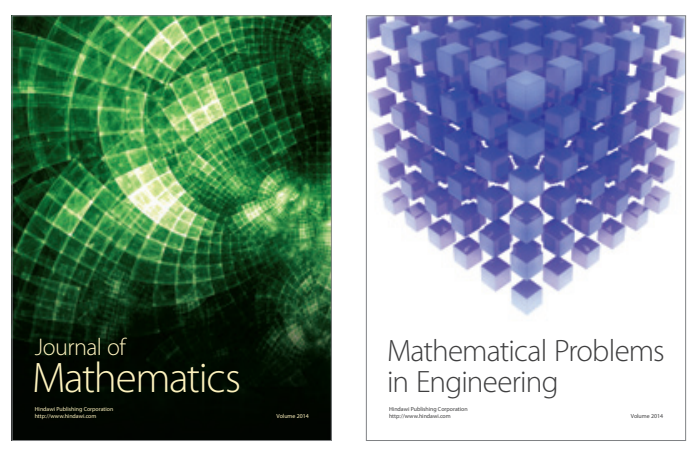

Mathematical Problems in Engineering
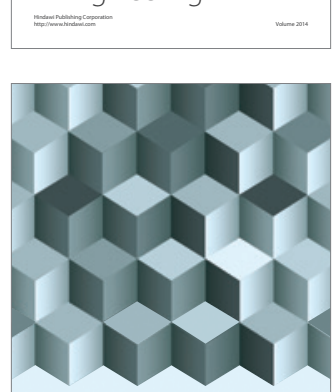

Journal of

Function Spaces
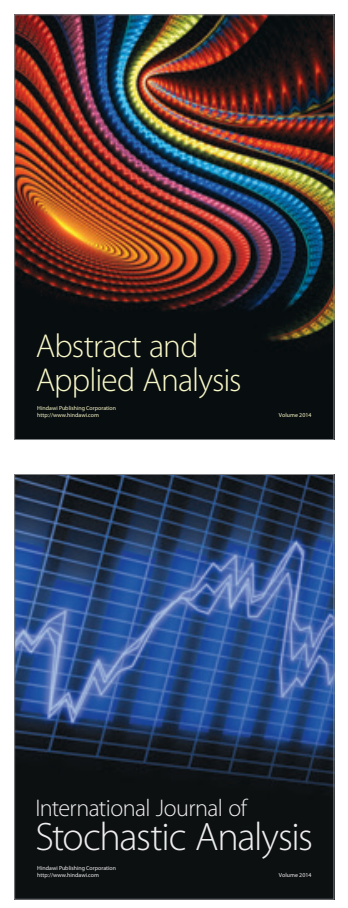

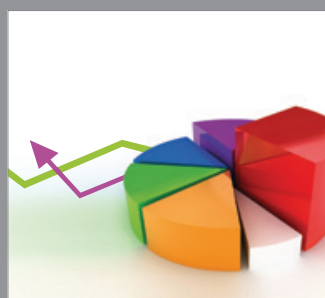

ournal of

Probability and Statistics

Promensencen
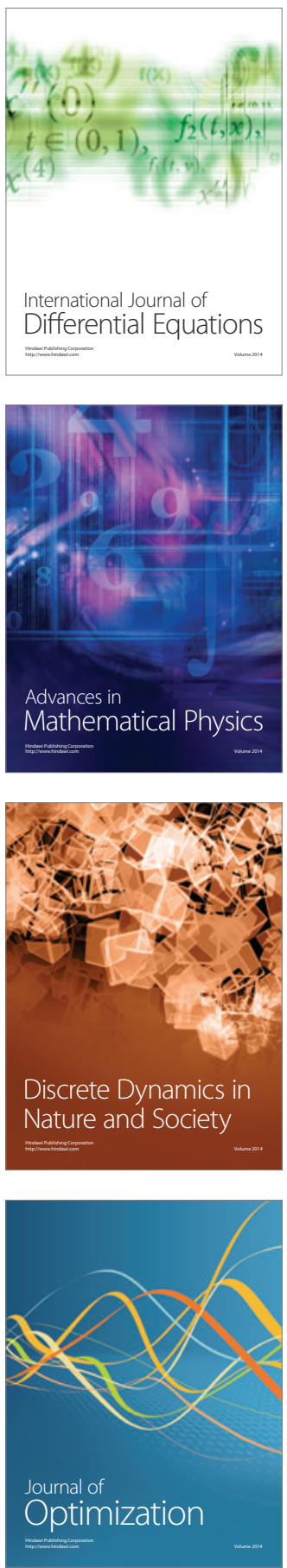